\title{
Higuchi bound on slow-roll inflation and the swampland
}

\author{
Marvin Lüben ${ }^{a}$ and Dieter Lüst ${ }^{a, b}$ \\ ${ }^{a}$ Max-Planck-Institut für Physik (Werner-Heisenberg-Institut), \\ Föhringer Ring 6, 80805 Munich, Germany \\ ${ }^{b}$ Arnold-Sommerfeld-Center for Theoretical Physics, Ludwig-Maximilians-Universität, \\ Theresienstr. 37, 80333 München, Germany \\ E-mail: mlueben@mpp.mpg.de, dieter.luest@lmu.de
}

\begin{abstract}
In this paper we study the implications of the generalized Higuchi bound on massive spin-two fields for the derivative of the scalar potential within bimetric theory. In contrast to the recent de Sitter swampland conjecture, an upper bound on the derivate of the scalar potential follows from the generalized Higuchi bound. In combination, this leaves a window for the derivate of the scalar potential. We discuss this bound in several representative bimetric models and parameter regions.
\end{abstract}

Keywords: Cosmology of Theories beyond the SM, Effective Field Theories, Superstring Vacua

ARXIV EPRINT: 2003.10494 


\section{Contents}

1 Introduction 1

2 Ingredients from bimetric theory $\quad 2$

2.1 Action and equations of motion 2

2.2 Proportional background solutions 3

2.3 Flat FLRW solutions 4

2.4 Generalized Higuchi bound 5

3 Constraint on the derivative of the scalar potential $\quad 6$

$\begin{array}{lll}4 & \text { Summary and discussion } & 7\end{array}$

$\begin{array}{ll}\text { A } \text { Model-specific considerations } & 9\end{array}$

\section{Introduction}

As it is extensively discussed during the recent two years, the effective potential of scalar fields in effective gravitational theories is severely constrained by fundamental quantum gravity or string theory considerations [1-6]. These constraints have profound implications on the evolution of the universe, in particular on inflationary scenarios and on the problem of dark energy $[7,8]$.

A genuine feature of quantum gravity and string theory is the existence of higher spin fields. The effective field theory of higher spin fields is highly constrained. One such constraint is the Higuchi bound [9],

$$
m^{2} \geq s(s-1) H^{2}
$$

for a massive (bosonic) field of spin $s$ and mass $m$ that propagats in de Sitter (dS) spacetime with radius $1 / H$ (with $H$ the Hubble parameter). If the bound is violated the massive higher spin field contains helicity modes with negative norm which is in conflict with unitarity. Recently some implications from the Higuchi bound on massive higher spin states in string theory and on the scale of inflation were discussed in [10-12].

The Higuchi bound is non-trivial for $s \geq 2$. In this article we focus on massive spin2 fields $[13,14]$ with Fierz-Pauli mass $m_{\mathrm{FP}}$. Here the Higuchi bound explicitly reads $m_{\mathrm{FP}}^{2} \geq 2 H^{2}$. In the case where the Hubble parameter is given by the potential energy of a scalar field, i.e. $3 H^{2}=V$, the Higuchi bound provides an upper limit on the potential energy as

$$
3 m_{\mathrm{FP}}^{2} \geq 2 V
$$

given the mass of the spin-2 field. 
In ref. [15] the Higuchi bound of dS spacetime was generalized for FLRW spacetime to a cosmological stability bound. This bound was found in the context of bimetric theory [16, 17] which serves as the low-energy effective field theory for a massive and a massless spin-2 field with fully non-linear (self-)interactions. In section 3 we examine implications of this bound in bimetric theory for the derivative of the scalar potential, denoted by $V^{\prime}$. In contrast to the swampland condition of [2], where a lower bound on $V^{\prime}$ is conjectured, we will show that an upper bound on $V^{\prime}$ follows from the generalized Higuchi bound.

\section{Ingredients from bimetric theory}

In order to describe a massive spin-2 field propagating on a curved background such as FLRW, the linear theory by Fierz and Pauli [13, 14, 18, 19] must be completed by nonlinear terms [20-22]. Bimetric theory is the ghost-free non-linear completion of Fierz-Pauli describing a gravitating massive spin-2 field [16, 17, 23, 24]. For a review we refer to ref. [25]. Furthermore, for some work about bimetric theory in relation to the swampland and string theory realizations see [26-30].

\subsection{Action and equations of motion}

We focus on singly-coupled bimetric theory where the Standard Model couples to only one of the metric tensors, say $g_{\mu \nu}$. The action for the metric tensors $g_{\mu \nu}$ and $f_{\mu \nu}$ is given by [16]

$$
\begin{aligned}
S= & \frac{M_{p}^{2}}{2} \int \mathrm{d}^{4} x\left(\sqrt{-g} R(g)+\alpha^{2} \sqrt{-f} R(f)\right) \\
& -M_{p}^{2} \int \mathrm{d}^{4} x \sqrt{-g} \mathcal{U}(g, f)+\int \mathrm{d}^{4} x \sqrt{-g} \mathcal{L}_{\mathrm{m}}\left(g_{\mu \nu}, \Phi\right),
\end{aligned}
$$

where $R(g)$ and $R(f)$ are the Ricci scalars of $g_{\mu \nu}$ and $f_{\mu \nu}$, resp. The quantity $M_{p}$ is the Planck mass of $g_{\mu \nu}$ and $\alpha=M_{f} / M_{p}$ measures its ratio to the Planck mass of $f_{\mu \nu}$. The bimetric potential $\mathcal{U}(g, f)$ is given in terms of the square-root matrix $S^{\mu}{ }_{\nu}$ defined by the relation

$$
S_{\alpha}^{\mu} S_{\nu}^{\alpha}=g^{\mu \alpha} f_{\alpha \nu}
$$

which in general does not have an unique solution. Only if the metrics $g_{\mu \nu}$ and $f_{\mu \nu}$ share the same time- and space-like directions, the theory is defined unambiguously and an unique and real solution to eq. (2.2) exists [31]. Then, the bimetric potential reads

$$
\mathcal{U}(g, f)=\sum_{n=0}^{4} \beta_{n} e_{n}(S)
$$

in terms of the elementary symmetric polynomials $e_{n}$. The parameters $\beta_{n}$ are real and have mass dimension 2 in this parametrization. Note that $\beta_{0}$ and $\beta_{4}$ parametrize the vacuum energy in the $g_{\mu \nu^{-}}$and $f_{\mu \nu}$-sector, resp. Finally, Standard Model fields are collectively denoted as $\Phi$. 
Varying the action (2.1) with respect to $g^{\mu \nu}$ and $f^{\mu \nu}$ yields two sets of modified Einstein equations,

$$
G_{\mu \nu}^{g}+\mathcal{U}_{\mu \nu}^{g}=\frac{1}{M_{p}^{2}} T_{\mu \nu}, \quad \alpha^{2} G_{\mu \nu}^{f}+\mathcal{U}_{\mu \nu}^{f}=0
$$

where $G_{\mu \nu}^{g}$ and $G_{\mu \nu}^{f}$ are the Einstein tensors of $g_{\mu \nu}$ and $f_{\mu \nu}$, resp. The explicit form of contributions coming from the bimetric potential $\mathcal{U}_{\mu \nu}^{g}$ and $\mathcal{U}_{\mu \nu}^{f}$ can be found, e.g., in refs. $[16,32]$. The stress-energy tensor of matter is defined as

$$
T_{\mu \nu}=\frac{-2}{\sqrt{-g}} \frac{\partial \sqrt{-g} \mathcal{L}_{\mathrm{m}}}{\partial g^{\mu \nu}}
$$

If the matter-sector $\sqrt{-g} \mathcal{L}_{\mathrm{m}}$ is invariant under diffeomorphisms, stress-energy is conserved, $\nabla^{\mu} T_{\mu \nu}=0$, where $\nabla_{\mu}$ is the covariant derivative compatible with $g_{\mu \nu}$. The Bianchi identity $\nabla^{\mu} G_{\mu \nu}^{g}=0$ implies the so-called Bianchi constraint

$$
\nabla^{\mu} \mathcal{U}_{\mu \nu}^{g}=0
$$

\subsection{Proportional background solutions}

An important class of solutions are the proportional backgrounds where both metric are related by a conformal factor, $f_{\mu \nu}=c^{2} g_{\mu \nu}$ [33]. The Bianchi constraint forces $c$ to be a constant. These solutions are maximally symmetric, i.e. (A)dS or Minkowski. The effective cosmological constant in terms of the bimetric parameters is given by

$$
\begin{aligned}
\Lambda & =V+\beta_{0}+3 \beta_{1} c+3 \beta_{2} c^{2}+\beta_{3} c^{3} \\
& =\frac{1}{\alpha^{2} c^{2}}\left(\beta_{1} c+3 \beta_{2} c^{2}+3 \beta_{3} c^{3}+\beta_{4} c^{4}\right) .
\end{aligned}
$$

The equality of the first and second line follows from the proportionality of the Einstein tensors for $g_{\mu \nu}$ and $f_{\mu \nu}$. Here, we already anticipated that the matter sector contributes potential energy $V$ to the effective cosmological constant. Therefore $\Lambda$ is composed out of vacuum energy, interaction energy of the massive spin-2 fields, and potential energy from the matter sector. ${ }^{1}$ Eq. (2.7) can be thought of as determining the proportionality constant $c$.

Bimetric theory has a well-defined mass spectrum around proportional backgrounds. The metric fluctuations are a linear superposition of mass eigenstates. The mass of the massive spin-2 field is given by

$$
m_{\mathrm{FP}}^{2}=\frac{1+\alpha^{2} c^{2}}{\alpha^{2} c^{2}} c\left(\beta_{1}+2 \beta_{2} c+\beta_{3} c^{2}\right)
$$

in terms of the bimetric parameters. We refer to this quantity as Fierz-Pauli mass. As mentioned previously, the Fierz-Pauli mass is subject to the Higuchi bound [9],

$$
3 m_{\mathrm{FP}}^{2}>2 \Lambda
$$

to ensure unitarity.

\footnotetext{
${ }^{1}$ Note that the vacuum energy contribution $\beta_{0}$ is degenerate with an unspecified potential $V$. In the analysis of this paper however, $V$ serves as an input parameter while the interaction parameters are fixed in terms of $V, m_{\mathrm{FP}}$ and $c$. Later we will go to the parameter regime where $\Lambda \simeq V$ or equivalently $V \gg \beta_{0}+3 \beta_{1} c+3 \beta_{2} c^{2}+\beta_{3} c^{3}$. For these reasons we treat $V$ and $\beta_{0}$ as independent parameters.
} 


\subsection{Flat FLRW solutions}

Assuming homogeneity and isotropy according to the cosmological principle, both metrics are of FLRW from [34-36],

$$
\begin{aligned}
\mathrm{d} s_{g}^{2} & =-\mathrm{d} t^{2}+a^{2} \mathrm{~d} \vec{x}^{2} \\
\mathrm{~d} s_{f}^{2} & =-X^{2} \mathrm{~d} t^{2}+b^{2} \mathrm{~d} \vec{x}^{2} .
\end{aligned}
$$

Here, the functions $a$ and $b$ are the scale factors of $g_{\mu \nu}$ and $f_{\mu \nu}$, resp., while $X$ is the lapse of $f_{\mu \nu}$. All metric functions depend on time $t$ only. We introduce the Hubble rate $H$ and the scale factor ratio $y$ as

$$
H=\frac{\dot{a}}{a}, \quad y=\frac{b}{a},
$$

where a dot denotes derivative with respect to cosmic time $t$.

Next, we assume that the matter sector consists of a real scalar field $\phi$ with Lagrangian

$$
\mathcal{L}_{\mathrm{m}}=-\frac{1}{2} g^{\mu \nu} \partial_{\mu} \phi \partial_{\nu} \phi-V(\phi)
$$

with scalar potential $V(\phi)$. According to the cosmological principle, the scalar field can only depend on time $t$. Consequently, the energy density and pressure are given by

$$
\frac{\rho_{\phi}}{M_{p}^{2}}=\frac{1}{2} \dot{\phi}^{2}+V, \quad \frac{p_{\phi}}{M_{p}^{2}}=\frac{1}{2} \dot{\phi}^{2}-V
$$

The stress-energy tensor is given by

$$
T_{\nu}^{\mu}=\operatorname{diag}\left(-\rho_{\phi}, p_{\phi}, p_{\phi}, p_{\phi}\right) .
$$

The equation of motion for the scalar field reads

$$
\ddot{\phi}+3 H \dot{\phi}+V_{\phi}=0 .
$$

Next, we derive the Friedmann equations. The Bianchi constraint is solved by

$$
X \dot{a}=\dot{b},
$$

on the so-called dynamical branch. ${ }^{2}$ Plugging the ansatz (2.10) into the modified Einstein eq. (2.4) yields two sets of Friedmann equations. These can be rearranged to give a single Friedmann equation. Here, we adopt the notation of ref. [39] where the Friedmann equation reads

$$
3 H^{2}=\frac{\rho_{\phi}}{M_{\text {eff }}^{2}}
$$

\footnotetext{
${ }^{2}$ In principle, another solution to the Bianchi constraint exists. On this algebraic branch, $y$ is forced to be a constant and the solution has various pathologies [37, 38].
} 
in terms of the time-varying effective Planck mass

$$
M_{\mathrm{eff}}^{2}=\frac{1+\alpha^{2} y^{2}}{1+\frac{U}{\rho_{\phi}}} M_{p}^{2} .
$$

The function $U$ depends on the parameters of the bimetric potential as

$$
U=M_{p}^{2}\left(\beta_{0}+4 \beta_{1} y+6 \beta_{2} y^{2}+4 \beta_{3} y^{3}+\beta_{4} y^{4}\right)
$$

The scale factor ratio is determined by a quartic polynomial,

$$
P(y)=\rho_{\phi},
$$

where the polynomial $P(y)$ is given by

$$
P(y)=\frac{1+\alpha^{2} y^{2}}{4 \alpha^{2} y} U^{\prime}-U
$$

in terms of $U$ with $U^{\prime}=\mathrm{d} U / \mathrm{d} y$. The consistency of the solutions to this equation have been studied in detail in refs. [40-42]. However, in this note we will adopt a perturbative treatment.

\subsection{Generalized Higuchi bound}

By inspecting the sign of the kinetic term of the scalar mode in the minisuperspace approximation, the authors of ref. [15] derived a cosmological stability bound that generalizes the Higuchi bound to FLRW spacetime. Let us define a time dependent mass parameter as

$$
m_{\text {gen }}^{2}=\frac{1+\alpha^{2} y^{2}}{\alpha^{2} y}\left(\beta_{1}+2 \beta_{2} y+\beta_{3} y^{2}\right)
$$

This quantity reduces to the Fierz-Pauli mass (2.8) when the metrics are proportional, ${ }^{3}$ i.e., for $y=X=c$. It was shown that the kinetic term of the scalar mode is multiplied by

$$
W=m_{\text {gen }}^{2}-2 H^{2}
$$

The kinetic term of the scalar mode has the correct sign if

$$
W>0
$$

This is the generalized Higuchi bound that we will use in the next section. Note, that this bound reduces to the standard Higuchi bound when the metrics are proportional.

\footnotetext{
${ }^{3}$ Note, that a well-defined notion of mass in the representation-theoretic sense does not exist in FLRW. In order to define a mass, the background spacetime needs to satisfy Lorentz- or (A)dS-isometries.
} 


\section{Constraint on the derivative of the scalar potential}

As mentioned previously, the Higuchi bound provides an upper limit on the (local) minimum of the scalar potential. By studying small displacements from the minimum, we now derive a bound on the derivative of the potential close to its minimum. We will work in the slow-roll approximation. We assume that $\Lambda \simeq V$, i.e., the potential energy of the scalar field dominates over the vacuum and interaction energy. We further assume that the Higuchi bound is not saturated, i.e., we exclude the partially-massless case [43, 44], in order to avoid devision by zero.

First, we express the energy density of the scalar field in terms of the potential $V$ and its derivative. Let us introduce the slow-roll parameter

$$
\epsilon=\frac{M_{\mathrm{eff}}^{2}}{2}\left(\frac{V^{\prime}}{V}\right)^{2}
$$

where $V^{\prime}$ means derivative of $V$ with respect to the scalar field $\phi$. In the slow-roll approximation $\epsilon \ll 1$ and $\ddot{\phi} \ll H \dot{\phi}$, the scalar equation of motion (2.15) can be rearranged to

$$
\dot{\phi}=-\frac{V^{\prime}}{3 H}=-\frac{\sqrt{2 \epsilon} V}{3 M_{\mathrm{eff}} H} .
$$

Replacing $H$ using the Friedmann eqs. (2.13) and (2.17) yields after rearranging

$$
\left(\frac{1}{2} \dot{\phi}^{2}+V\right) \dot{\phi}^{2}=\frac{2 \epsilon}{3} V^{2}
$$

Solving for $\dot{\phi}$ yields $\dot{\phi}^{2}=-V(1 \pm \sqrt{1+4 \epsilon / 3})$ out of which only the '-' branch is physical (as only then $\dot{\phi}=0$ for $\epsilon=0$ ). Plugging this back into our expression for the energy density yields

$$
\frac{\rho_{\phi}}{M_{p}^{2}}=V\left(1+\frac{\epsilon}{3}\right)
$$

up to first order in slow-roll. Plugging this into the Friedmann equation yields

$$
3 H^{2}=\frac{\rho_{\phi}}{M_{\mathrm{eff}}^{2}}=V\left(1+\frac{\epsilon}{3}\right) .
$$

Here we used that $M_{\text {eff }} / M_{p} \sim \mathcal{O}(1)$ during slow-roll.

To find an expression for the generalized spin-2 mass $m_{\text {gen }}$ up to first order in slow-roll we plug eq. (3.4) into the quartic polynomial (2.20) and solve for $y$ order by order in $\epsilon$. This results in

$$
y=c\left(1-\frac{V}{3 m_{\mathrm{FP}}^{2}-2 V} \frac{\epsilon}{3}\right) .
$$

Here, $c$ is the solution for $\epsilon=0$, i.e. at the de Sitter point where the cosmological constant is given by the potential energy of the scalar field only. The Fierz-Pauli mass is defined on that background as in eq. (2.8). Plugging this into eq. (2.22) yields

$$
m_{\mathrm{gen}}^{2}=m_{\mathrm{FP}}^{2}+F \frac{\epsilon}{3}
$$


up to first order in slow-roll. The quantity $F$ depends on the bimetric parameters and scalar potential as

$$
F=-\frac{V c}{3 m_{\mathrm{FP}}^{2}-2 V}\left(\frac{\beta_{3} c^{2}-\beta_{1}}{\alpha^{2} c^{2}}+\beta_{1}+4 \beta_{2} c+3 \beta_{3} c^{2}\right) .
$$

The expression in parenthesis does not combine into well-defined background quantities.

Combining our ingredients, we find $W$ in the slow-roll approximation as

$$
0 \leq W=3 m_{\mathrm{FP}}^{2}-2 V+\left(F-\frac{2}{3} V\right) \epsilon .
$$

For $F \geq 2 V / 3$ the bound on $\epsilon$ is trivial. However, if the Higuchi bound is satisfied and if $F<2 V / 3$, we find the following non-trivial bound on $\epsilon$ :

$$
\epsilon \leq 3 \frac{3 m_{\mathrm{FP}}^{2}-2 V}{2 V-3 F}
$$

In appendix A we discuss typical values of the right hand side of eq. (3.9) by studying various simplified models and different regions of the parameter space. We generally find that the right hand side is of the order of $m_{\mathrm{FP}}^{2} / V$. That means, if the mass of the spin-2 field is close to the Higuchi bound, the restriction on $V^{\prime}$ is most stringent as we assumed slow-roll to start with.

Finally, the bound (3.9) implies for the derivative of the scalar potential,

$$
\frac{\left|V^{\prime}\right|}{V} \lesssim \frac{\sqrt{6}}{M_{p}} \sqrt{\frac{3 m_{\mathrm{FP}}^{2}-2 V}{2 V-3 F}} .
$$

Here we used again that generally $M_{\text {eff }} / M_{p} \sim \mathcal{O}(1)$. Hence, we found an upper limit on the absolute value of the derivative of the scalar potential. The restriction is most stringent close to the Higuchi bound. Saturation of the bound is excluded and hence the right hand side cannot vanish.

\section{Summary and discussion}

We used bimetric theory as the low-energy effective field theory to describe a massive spin-2 field with mass $m_{\mathrm{FP}}$ propagating on FLRW spacetime. Assuming that the matter sector is composed out of a scalar field with potential $V$, we showed that the generalized Higuchi bound implies an upper bound on the slow-roll parameter of the following form:

$$
\epsilon \leq c \frac{m_{\mathrm{FP}}^{2}}{V}, \quad \text { for } c \sim \mathcal{O}(1) .
$$

For the derivative of the scalar potential an equivalent upper bound can be written as

$$
\frac{\left|V^{\prime}\right|}{V} \leq \frac{c}{M_{p}} \sqrt{\frac{m_{\mathrm{FP}}^{2}}{V}} .
$$


In our derivation we assumed that only the potential energy of the scalar field contributes to an effective cosmological constant. For a large spin-2 mass $m_{\mathrm{FP}}^{2} \gg V$, this bound is not restrictive as we assumed slow-roll (i.e. $\epsilon \ll 1$ ) in our derivation. On the other hand, if the mass of the spin-2 field is close to the Higuchi bound, the derivative of the scalar potential is bounded from above.

Let us compare our result with the bounds on the scalar potential, which are obtained in the context of the de Sitter swampland conjecture $[2,4]$, stating that

$$
\frac{\left|V^{\prime}\right|}{V} \geq \frac{c^{\prime}}{M_{p}} \quad \text { or } \quad V^{\prime \prime} \leq-\frac{c^{\prime \prime}}{M_{p}^{2}} V
$$

with $c^{\prime}, c^{\prime \prime}$ being some universal constants of order one. This bound rules out de Sitter vacua and restricts slow-roll inflationary scenarios in string theory.

Applying the lower bound from the de Sitter swampland conjecture and combining it with the upper bound from the generalized Higuchi bound in bimetric theory, it follows that the derivative of the scalar potential has to lie in the following window:

$$
\frac{c^{\prime}}{M_{p}} \leq \frac{\left|V^{\prime}\right|}{V} \leq \frac{c}{M_{p}} \sqrt{\frac{m_{\mathrm{FP}}^{2}}{V}} .
$$

The window is small when the spin-2 mass is close to the Higuchi bound, in which case the derivative has to be of the order $\left|V^{\prime}\right| / V \simeq 1 / M_{p}$. However, due to the Higuchi bound, the window does not close completely. Let us point out some caveats again. The Higuchi bound on $V^{\prime}$ was found within the slow-roll approximation. Further, there are specific parameter regions where $V^{\prime}$ is not bounded from above. However, if the bound in eq. (4.4) on $V^{\prime}$ is respected the generalized Higuchi bound is guaranteed to be satisfied for generic parameter values.

In string theory it is natural to assume that the spin- 2 mass $m_{\mathrm{FP}}$ is related to higher spin string excitations [27]. Assuming that eq. (4.2) holds generally and identifying the spin-2 mass with the string scale, $m_{\mathrm{FP}} \simeq M_{s}$, our bound reads

$$
\frac{\left|V^{\prime}\right|}{V} \leq c \frac{M_{s}}{M_{p}} \sqrt{\frac{1}{V}} \simeq g_{s} \sqrt{\frac{1}{V \mathcal{V}}} .
$$

Here $g_{s}$ is the string coupling constant, and $\mathcal{V}$ denotes the internal volume of the compact six-dimensional space. We can use the bound on $M_{s}$ that was obtained in ref. [10],

$$
M_{s}^{2} \geq \sqrt{\frac{V}{3}} M_{p}
$$

which follows when one requires that the entire string Regge trajectory satisfied the Higuchi bound. So taking also this bound into account, the condition (4.5) is always satisfied if the following stronger bound holds:

$$
\frac{\left|V^{\prime}\right|}{V} \leq \frac{c}{M_{p}} \sqrt{\frac{M_{p}}{(V / 3)^{1 / 2}}} .
$$


As a next step it would be interesting to study whether the upper bound on $V^{\prime}$ is a generic implication of unitarity when massive spin-2 fields propagate on dS spacetime. This requires a treatment beyond slow-roll. Further, it would be interesting to check, wether this combined bound is satisfied, e.g., in concrete string theory settings.

\section{Acknowledgments}

We are grateful to E. Palti for useful discussions. This work is supported by a grant from the Max-Planck-Society. The work of D.L. is supported by the Origins Excellence Cluster.

\section{A Model-specific considerations}

In this section, we provide explicit expressions for the upper bound on $\epsilon$ in terms of the background variables $m_{\mathrm{FP}}$ and $V$ for several representative bimetric models and parameter regions. The relation between the interaction parameters $\beta_{n}$ and the background quantities is determined by eqs. (2.7) and (2.8) as described in detail in ref. [42]. Note that in general, the bimetric potential gives rise to an effective cosmological constant $\Lambda_{\text {bim }}=\beta_{0}+3 \beta_{1} c+3 \beta_{2} c^{2}+\beta_{3} c^{3}$. Here, we assume that only the scalar potential contributes to the overall cosmological constant, i.e. we assume $\Lambda \simeq V$ and the contributions from vacuum energy and interaction energy are subdominant, i.e., $V \gg \Lambda_{\text {bim }}$.

In the following, we only state the results and point out some caveats about the more restricted submodels. For brevity, let us define the maximum value of $\epsilon$ as

$$
\epsilon_{\max }=\frac{3\left(3 m_{\mathrm{FP}}^{2}-2 V\right)}{2 V-3 F},
$$

in terms of which the generalized Higuchi bound in slow-roll approximation reads $\epsilon<\epsilon_{\max }$.

Before studying individual models we point out again that an unspecified scalar potential $V$ is degenerate with the vacuum energy parameter $\beta_{0}$. In our analysis however, the potential $V$ is not unspecified. Instead, from the Higuchi bound we derive an upper limit on $\left|V^{\prime}\right|$ given the values of $m_{\mathrm{FP}}$ and $V$. The interaction parameters $\beta_{n}$, including $\beta_{0}$, are determined by eqs. (2.7) and (2.8) in terms of $m_{\mathrm{FP}}$ and $V$.

Setting $\beta_{2}=\beta_{3}=\beta_{4}=0$ in eqs. (2.7) and (2.8), solving for $\beta_{0}$ and $\beta_{1}$, and plugging the results into eq. (A.1) we find

$$
\epsilon_{\max }^{01}=\frac{3\left(3 m_{\mathrm{FP}}^{2}-2 V\right)^{2}}{\left(3 m_{\mathrm{FP}}^{2}-4 V\right) V}
$$

as the upper limit on $\epsilon$ in the $\beta_{0} \beta_{1}$-model. ${ }^{4}$ This model is only well-defined for $m_{\mathrm{FP}}^{2}>$ $V$ which is more restrictive than the Higuchi bound [42]. Spin-2 masses in the range $V<m_{\mathrm{FP}}^{2}<\frac{4}{3} V$ imply $\epsilon_{\max }^{01}<0$. Since $\epsilon$ is a manifestly positive quantity, the bound is trivial in this parameter region. Expanding around $m_{\mathrm{FP}}^{2} \gg V$ the expression simplifies to $\epsilon_{\max }^{01} \simeq 9 m_{\mathrm{FP}}^{2} / V$. Next, for the $\beta_{1} \beta_{4}$-model, we set $\beta_{0}=\beta_{2}=\beta_{3}=0$, solve eqs. (2.7)

\footnotetext{
${ }^{4}$ Here we keep $\beta_{0}$ as a free parameter. Setting $\beta_{0}$ to zero or absorbing the parameter into $V$ fixes the spin- 2 mass to be $m_{\mathrm{FP}}^{2}=V$. Keeping $\beta_{0}$ and $V$ independent allows $m_{\mathrm{FP}}$ to be independent.
} 
and (2.8) for $\beta_{1}$ and $\beta_{4}$ and plug the result into eq. (A.1). This leads to the same expression as for the $\beta_{0} \beta_{1}$-model, $\epsilon_{\max }^{14}=\epsilon_{\max }^{01}$. Therefore, close to the Higuchi bound the restriction on $\epsilon$ is most stringent. Moving to the $\beta_{1} \beta_{2}$-model by setting $\beta_{0}=\beta_{3}=\beta_{4}=0$ the same procedure leads to

$$
\epsilon_{\max }^{12}=\frac{3\left(3 m_{\mathrm{FP}}^{2}-V\right)^{2}}{\left(15 m_{\mathrm{FP}}^{2}-7 V\right) V}
$$

In the limit of large spin-2 mass we can approximate the bound as $\epsilon_{\max }^{12} \simeq 9 m_{\mathrm{FP}}^{2} /(5 V)$. Finally for the $\beta_{1} \beta_{3}$-model we set $\beta_{0}=\beta_{2}=\beta_{4}=0$ to find

$$
\epsilon_{\max }^{13}=\frac{3\left(3 m_{\mathrm{FP}}^{2}-2 V\right)^{2}}{16\left(3 m_{\mathrm{FP}}^{2}-V\right) m_{\mathrm{FP}}^{2}} .
$$

Expanding for $m_{\mathrm{FP}}^{2} \gg V$ this expression reduces to $\epsilon_{\max }^{13} \simeq 9 / 16$. Therefore, the upper limit on $\epsilon$ in the $\beta_{1} \beta_{3}$-model differs significantly from the upper limit in the other two parameter models.

Summarizing, for the two parameter models we find that $\epsilon_{\max } \simeq m_{\mathrm{FP}}^{2} / V$ in general. Only the $\beta_{1} \beta_{3}$-model is an exception, which implies the stronger bound $\epsilon_{\max } \leq 9 / 16$.

Next, we study more general cases by moving to three parameter models. We focus on the $\beta_{0} \beta_{1} \beta_{4}$-model with vacuum energy for $g_{\mu \nu}$ and for $f_{\mu \nu}$ and on the $\beta_{1} \beta_{2} \beta_{3}$-model without vacuum energy. For convenience, we introduce the quantity $\bar{\alpha}=\alpha c$ [42]. For the $\beta_{0} \beta_{1} \beta_{4}$-model we set $\beta_{2}=\beta_{3}=0$, solve eqs. (2.7) and (2.8) for $\beta_{0}, \beta_{1}$ and $\beta_{4}$ and plug the result into eq. (A.1). This leads to the following upper limit on $\epsilon$,

$$
\epsilon_{\max }^{014}=\frac{3\left(3 m_{\mathrm{FP}}^{2}-2 V\right)^{2}}{\left(3 m_{\mathrm{FP}}^{2}-4 V\right) V},
$$

which coincides with the expression that we obtained for the $\beta_{0} \beta_{1}$ - and $\beta_{1} \beta_{4}$-models. Moving to the $\beta_{1} \beta_{2} \beta_{3}$-model by setting $\beta_{0}=\beta_{4}=0$, the same procedure leads to

$$
\epsilon_{\max }^{123}=\frac{6\left(3 m_{\mathrm{FP}}^{2}-2 V\right)^{2}}{\left(12 m_{\mathrm{FP}}^{2}-\left(5-3 \bar{\alpha}^{2}\right) V\right) V} .
$$

In figure 1 we plot $\epsilon_{\max }^{123}$ as a function of $m_{\mathrm{FP}}^{2} / V$ for different values of $\bar{\alpha}$. For $\bar{\alpha} \lesssim 1$, the expression for $\epsilon_{\max }^{123}$ becomes independent of $\bar{\alpha}$ and can be approximated by $m_{\mathrm{FP}}^{2} / V$. For larger values of $\bar{\alpha}$, the upper limit on $\epsilon$ is suppressed by inverse powers of $\bar{\alpha}$. This can be understood from expanding eq. (A.6) in different limits. For $m_{\mathrm{FP}}^{2} \gg V$ the expression reduces to $\epsilon_{\max }^{123} \simeq 9 m_{\mathrm{FP}}^{2} /(2 V)$ which is independent of $\bar{\alpha}$. Expanding for $\bar{\alpha} \gg 1$ we find $\epsilon_{\max }^{123} \simeq 2\left(9 m_{\mathrm{FP}}^{4} / V^{2}-12 m_{\mathrm{FP}}^{2} / V+4\right) / \bar{\alpha}^{2}$. Therefore, in this limit $\epsilon_{\max }^{123}$ is suppressed by inverse powers of $\bar{\alpha}$ and the leading term is proportional to $\left(m_{\mathrm{FP}}^{2} / V\right)^{2}$ in contrast to our previously studied scenarios. This parameter region however is excluded by consistency conditions [42]. In the limit $\bar{\alpha} \ll 1$, the upper limit reads $\epsilon_{\max }^{123} \simeq 6\left(3 m_{\mathrm{FP}}^{2}-2 V\right)^{2} /\left(\left(12 m_{\mathrm{FP}}^{2}-\right.\right.$ $5 V) V$ ) which is again independent of $\bar{\alpha}$.

Summarizing the results for the three parameter models, within the consistent parameter region we find the same order of magnitude for the upper limit on $\epsilon$ as for the two 


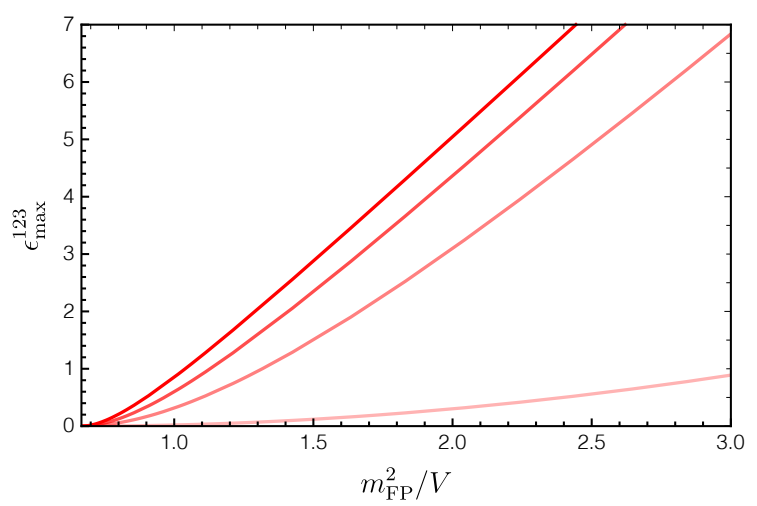

Figure 1. The upper limit on $\epsilon$ is shown as a function of $m_{\mathrm{FP}}^{2} / V$ for the $\beta_{1} \beta_{2} \beta_{3}$-model for different values of $\bar{\alpha}$. We chose the values $\bar{\alpha}=0.6,0.8,2,10$, where the lightest color corresponds to the largest value of $\bar{\alpha}$.

parameter models. That is $\epsilon_{\max } \sim m_{\mathrm{FP}}^{2} / V$. Only the $\beta_{1} \beta_{2} \beta_{3}$-model in the limit $\bar{\alpha} \gg 1$ is an exception. Instead of discussing also the other three parameter models individually, we now move to the full bimetric model with all interaction parameters left free.

With all interaction parameters left free, we solve the background eqs. (2.7) and (2.8) for the interaction parameters $\beta_{1}, \beta_{2}$, and $\beta_{3}$ in terms of the physical parameters $m_{\mathrm{FP}}$, $\Lambda=V$, and $\bar{\alpha}=\alpha c$. We also introduce the quantity $\bar{\beta}_{4}=\alpha^{-4} \beta_{4}$. In terms of these parameters, the upper limit on $\epsilon$ reads

$$
\epsilon_{\max }=\frac{6 \bar{\alpha}^{2}\left(3 m_{\mathrm{FP}}^{2}-2 V\right)^{2}}{\left(3 \beta_{0}+\bar{\alpha}^{2}\left(12 m_{\mathrm{FP}}^{2}-5 V+3 \beta_{0}\right)+3 \bar{\alpha}^{4}\left(V-\bar{\beta}_{4}\right)-3 \bar{\alpha}^{6} \bar{\beta}_{4}\right) V} .
$$

Although this expression is quite lengthy, we can discuss the order of magnitude for different parameter regimes. In the limit of large spin-2 mass, $m_{\mathrm{FP}}^{2} \gg V$, while keeping $\bar{\alpha}$ finite, the expression reduces to

$$
\epsilon_{\max } \simeq \frac{9}{2} \frac{m_{\mathrm{FP}}^{2}}{V}, \text { for } m_{\mathrm{FP}}^{2} \gg V,
$$

which is independent of $\bar{\alpha}$. Next, we expand $\epsilon_{\max }$ for small (GR-limit) and for large (massive gravity limit) $\bar{\alpha}$ resulting in

$$
\begin{aligned}
\epsilon_{\max } & =\frac{2 \bar{\alpha}^{2}\left(3 m_{\mathrm{FP}}^{2}-2 V\right)^{2}}{\beta_{0} V}, \text { for } \bar{\alpha} \ll 1 \\
\epsilon_{\max } & =-\frac{2\left(3 m_{\mathrm{FP}}^{2}-2 V\right)^{2}}{\bar{\alpha}^{4} \bar{\beta}_{4} V}, \text { for } \bar{\alpha} \gg 1 .
\end{aligned}
$$

In both limits, the order of magnitude is set by $m_{\mathrm{FP}}^{4} /\left(\beta_{n} V\right)$ and suppressed by (inverse) powers of $\bar{\alpha}$. However, this conclusion is model-dependent. In our parametrization the interaction parameters $\beta_{0}$ and $\bar{\beta}_{4}$ are fixed, while the other parameters scale with $\bar{\alpha}$ in a nontrivial way. We also studied the limit of small and large $\bar{\alpha}$ in other parametrizations. We find that $\epsilon_{\max }$ is not necessarily suppressed by (inverse) powers of $\bar{\alpha}$, while in these cases the order of magnitude is set by $m_{\mathrm{FP}}^{2} / V$. 
Summarizing, there are regions of the parameter space where the bound on $\epsilon$ is trivial either because $\epsilon_{\max } \gg 1$ or because $\epsilon_{\max }<0$. However, as the typical value we find $\epsilon_{\mathrm{max}} \simeq m_{\mathrm{FP}}^{2} / V$. Therefore, close to the Higuchi bound the limit on the gradient of the scalar potential is most stringent. In other words, for $\epsilon \lesssim m_{\mathrm{FP}}^{2} / V$ the Higuchi bound is guaranteed to be satisfied.

Open Access. This article is distributed under the terms of the Creative Commons Attribution License (CC-BY 4.0), which permits any use, distribution and reproduction in any medium, provided the original author(s) and source are credited.

\section{References}

[1] G. Dvali and C. Gomez, Quantum Compositeness of Gravity: Black Holes, AdS and Inflation, JCAP 01 (2014) 023 [arXiv: 1312.4795] [INSPIRE].

[2] G. Obied, H. Ooguri, L. Spodyneiko and C. Vafa, De Sitter Space and the Swampland, arXiv: 1806.08362 [INSPIRE].

[3] G. Dvali and C. Gomez, On Exclusion of Positive Cosmological Constant, Fortsch. Phys. 67 (2019) 1800092 [arXiv: 1806.10877] [InSPIRE].

[4] H. Ooguri, E. Palti, G. Shiu and C. Vafa, Distance and de Sitter Conjectures on the Swampland, Phys. Lett. B 788 (2019) 180 [arXiv:1810.05506] [INSPIRE].

[5] G. Dvali, C. Gomez and S. Zell, Quantum Breaking Bound on de Sitter and Swampland, Fortsch. Phys. 67 (2019) 1800094 [arXiv:1810.11002] [INSPIRE].

[6] E. Palti, The Swampland: Introduction and Review, Fortsch. Phys. 67 (2019) 1900037 [arXiv: 1903.06239] [INSPIRE].

[7] P. Agrawal, G. Obied, P.J. Steinhardt and C. Vafa, On the Cosmological Implications of the String Swampland, Phys. Lett. B $\mathbf{7 8 4}$ (2018) 271 [arXiv:1806.09718] [InSPIRE].

[8] S.K. Garg and C. Krishnan, Bounds on Slow Roll and the de Sitter Swampland, JHEP 11 (2019) 075 [arXiv: 1807.05193] [InSPIRE].

[9] A. Higuchi, Forbidden Mass Range for Spin-2 Field Theory in de Sitter Space-time, Nucl. Phys. B 282 (1987) 397 [INSPIRE].

[10] D. Lüst and E. Palti, A Note on String Excitations and the Higuchi Bound, Phys. Lett. B 799 (2019) 135067 [arXiv: 1907.04161] [INSPIRE].

[11] T. Noumi, T. Takeuchi and S. Zhou, String Regge trajectory on de Sitter space and implications to inflation, arXiv:1907.02535 [INSPIRE].

[12] M. Scalisi, Inflation, Higher Spins and the Swampland, Phys. Lett. B 808 (2020) 135683 [arXiv: 1912.04283] [INSPIRE].

[13] W. Pauli and M. Fierz, On Relativistic Field Equations of Particles With Arbitrary Spin in an Electromagnetic Field, Helv. Phys. Acta 12 (1939) 297 [InSPIRE].

[14] M. Fierz and W. Pauli, On relativistic wave equations for particles of arbitrary spin in an electromagnetic field, Proc. Roy. Soc. Lond. A A 173 (1939) 211 [inSPIRE].

[15] M. Fasiello and A.J. Tolley, Cosmological Stability Bound in Massive Gravity and Bigravity, JCAP 12 (2013) 002 [arXiv:1308.1647] [INSPIRE]. 
[16] S.F. Hassan and R.A. Rosen, Bimetric Gravity from Ghost-free Massive Gravity, JHEP 02 (2012) 126 [arXiv:1109.3515] [INSPIRE].

[17] S.F. Hassan and R.A. Rosen, Confirmation of the Secondary Constraint and Absence of Ghost in Massive Gravity and Bimetric Gravity, JHEP 04 (2012) 123 [arXiv:1111.2070] [INSPIRE].

[18] A.I. Vainshtein, To the problem of nonvanishing gravitation mass, Phys. Lett. B 39 (1972) 393 [INSPIRE].

[19] D.G. Boulware and S. Deser, Can gravitation have a finite range?, Phys. Rev. D 6 (1972) 3368 [INSPIRE].

[20] L. Bernard, C. Deffayet and M. von Strauss, Consistent massive graviton on arbitrary backgrounds, Phys. Rev. D 91 (2015) 104013 [arXiv:1410.8302] [InSPIRE].

[21] L. Bernard, C. Deffayet and M. von Strauss, Massive graviton on arbitrary background: derivation, syzygies, applications, JCAP 06 (2015) 038 [arXiv: 1504.04382] [INSPIRE].

[22] L. Bernard, C. Deffayet, A. Schmidt-May and M. von Strauss, Linear spin-2 fields in most general backgrounds, Phys. Rev. D 93 (2016) 084020 [arXiv:1512.03620] [InSPIRE].

[23] C. de Rham and G. Gabadadze, Generalization of the Fierz-Pauli Action, Phys. Rev. D 82 (2010) 044020 [arXiv: 1007.0443] [INSPIRE].

[24] C. de Rham, G. Gabadadze and A.J. Tolley, Resummation of Massive Gravity, Phys. Rev. Lett. 106 (2011) 231101 [arXiv:1011.1232] [INSPIRE].

[25] A. Schmidt-May and M. von Strauss, Recent developments in bimetric theory, J. Phys. A 49 (2016) 183001 [arXiv:1512.00021] [INSPIRE].

[26] C. Bachas and I. Lavdas, Massive Anti-de Sitter Gravity from String Theory, JHEP 11 (2018) 003 [arXiv: 1807.00591] [INSPIRE].

[27] S. Ferrara, A. Kehagias and D. Lüst, Bimetric, Conformal Supergravity and its Superstring Embedding, JHEP 05 (2019) 100 [arXiv: 1810.08147] [INSPIRE].

[28] D. Klaewer, D. Lüst and E. Palti, A Spin-2 Conjecture on the Swampland, Fortsch. Phys. 67 (2019) 1800102 [arXiv: 1811.07908] [inSPIRE].

[29] C. De Rham, L. Heisenberg and A.J. Tolley, Spin-2 fields and the weak gravity conjecture, Phys. Rev. D 100 (2019) 104033 [arXiv:1812.01012] [INSPIRE].

[30] C. Bachas, Massive AdS Supergravitons and Holography, JHEP 06 (2019) 073 [arXiv: 1905.05039] [INSPIRE].

[31] S.F. Hassan and M. Kocic, On the local structure of spacetime in ghost-free bimetric theory and massive gravity, JHEP 05 (2018) 099 [arXiv:1706.07806] [INSPIRE].

[32] S.F. Hassan and R.A. Rosen, On Non-Linear Actions for Massive Gravity, JHEP 07 (2011) 009 [arXiv: 1103.6055] [INSPIRE].

[33] S.F. Hassan, A. Schmidt-May and M. von Strauss, On Consistent Theories of Massive Spin-2 Fields Coupled to Gravity, JHEP 05 (2013) 086 [arXiv:1208.1515] [InSPIRE].

[34] M.S. Volkov, Cosmological solutions with massive gravitons in the bigravity theory, JHEP 01 (2012) 035 [arXiv:1110.6153] [INSPIRE].

[35] M. von Strauss, A. Schmidt-May, J. Enander, E. Mörtsell and S.F. Hassan, Cosmological Solutions in Bimetric Gravity and their Observational Tests, JCAP 03 (2012) 042 [arXiv:1111.1655] [INSPIRE]. 
[36] D. Comelli, M. Crisostomi, F. Nesti and L. Pilo, FRW Cosmology in Ghost Free Massive Gravity, JHEP 03 (2012) 067 [Erratum ibid. 06 (2012) 020] [arXiv:1111.1983] [InSPIRE].

[37] D. Comelli, M. Crisostomi and L. Pilo, Perturbations in Massive Gravity Cosmology, JHEP 06 (2012) 085 [arXiv: 1202.1986] [INSPIRE].

[38] G. Cusin, R. Durrer, P. Guarato and M. Motta, A general mass term for bigravity, JCAP 04 (2016) 051 [arXiv : 1512.02131] [INSPIRE].

[39] Y. Sakakihara and T. Tanaka, Primordial fluctuations from inflation in dRGT bimetric theory of gravity, JCAP 09 (2016) 033 [arXiv: 1605.05790] [INSPIRE].

[40] F. Koennig, A. Patil and L. Amendola, Viable cosmological solutions in massive bimetric gravity, JCAP 03 (2014) 029 [arXiv:1312.3208] [INSPIRE].

[41] F. Könnig, Higuchi Ghosts and Gradient Instabilities in Bimetric Gravity, Phys. Rev. D 91 (2015) 104019 [arXiv: 1503.07436] [INSPIRE].

[42] M. Lüben, A. Schmidt-May and J. Weller, Physical parameter space of bimetric theory and SN1 a constraints, arXiv:2003.03382 [INSPIRE].

[43] S. Deser and R.I. Nepomechie, Gauge Invariance Versus Masslessness in de Sitter Space, Annals Phys. 154 (1984) 396 [INSPIRE].

[44] S.F. Hassan, A. Schmidt-May and M. von Strauss, On Partially Massless Bimetric Gravity, Phys. Lett. B $\mathbf{7 2 6}$ (2013) 834 [arXiv: 1208.1797] [InSPIRE]. 